Acta Technologica Agriculturae 3

Nitra, Slovaca Universitas Agriculturae Nitriae, 2016, pp. 70-76

\title{
A REVIEW ON CURRENT AND EMERGING APPLICATION POSSIBILITIES FOR UNMANNED AERIAL VEHICLES
}

\author{
Ivan H. BELOEV
}

Angel Kanchev University of Ruse, Bulgaria

\begin{abstract}
This paper presents a review on current and emerging application possibilities for unmanned aerial vehicles (UAVs). The introduction section of the paper briefly describes some of the application areas in which drones are currently being used. The next chapters of the paper describe more detailly the use of UAVs for aerial photography, filming, security and logistics, GIS, land and water surveys. The main focus of the last chapters is on the advantages and the disadvantages of the drones usage in precision agriculture, wildlife and nature observations and archaeology. The last chapters also provide information on how the advanced information technology solutions can be implemented in order to provide means for fighting invasive species, to increase the yield of certain crops, to monitor and predict flooding, wildfires and other disasters, etc. This paper provides only overview of the most interesting and widely available applications of the UAVs, but there are also many other more specific and dedicated solutions for implementation of the drones for different purposes.
\end{abstract}

Keywords: unmanned aerial vehicles; aerial services; precision agriculture; forestry; safety applications

In the last few years, the unmanned aerial vehicles have been used for many different tasks and services. They are mostly used for aerial photographing and remote aerial reconnaissance. This is mainly because they can easily take an aerial image or record a video of a remote location and because they are especially suitable for use in the cases of difficult terrain or in hazardous or life threatening situations. The UAVs can be equipped with different high resolution cameras and according to the task, the obtained information can be streamed back in real time to a monitoring centre or stored locally on a flash card (Blyenburgh et al., 2011).

The UAVs can also be used for various scientific and research tasks - from remote observation of wildlife and monitoring of different natural occurrences to the sensing and the analysis of different environmental parameters (Lei
Ma et al., 2013). A different field of the UAVs application is in logistics and transportation, where the UAVs can be used to carry and deliver a variety of loads. The use of UAVs in the commercial services is a newly emerging trend in this field. Examples of some of the commercial tasks include the measurement of the extraction volume of different materials in quarries, measuring the amount of the timber volume and the number of stems in a forestry region, inspection of different crops for precise agriculture, examination or inspection of power lines and communication antennas and so on (DeBusk, 2009; Doherty and Rudol, 2007; Barnard, 2010; Manyoky et al., 2011). The purpose of this paper is to present some of the current and emerging services provided by unmanned aerial vehicles.
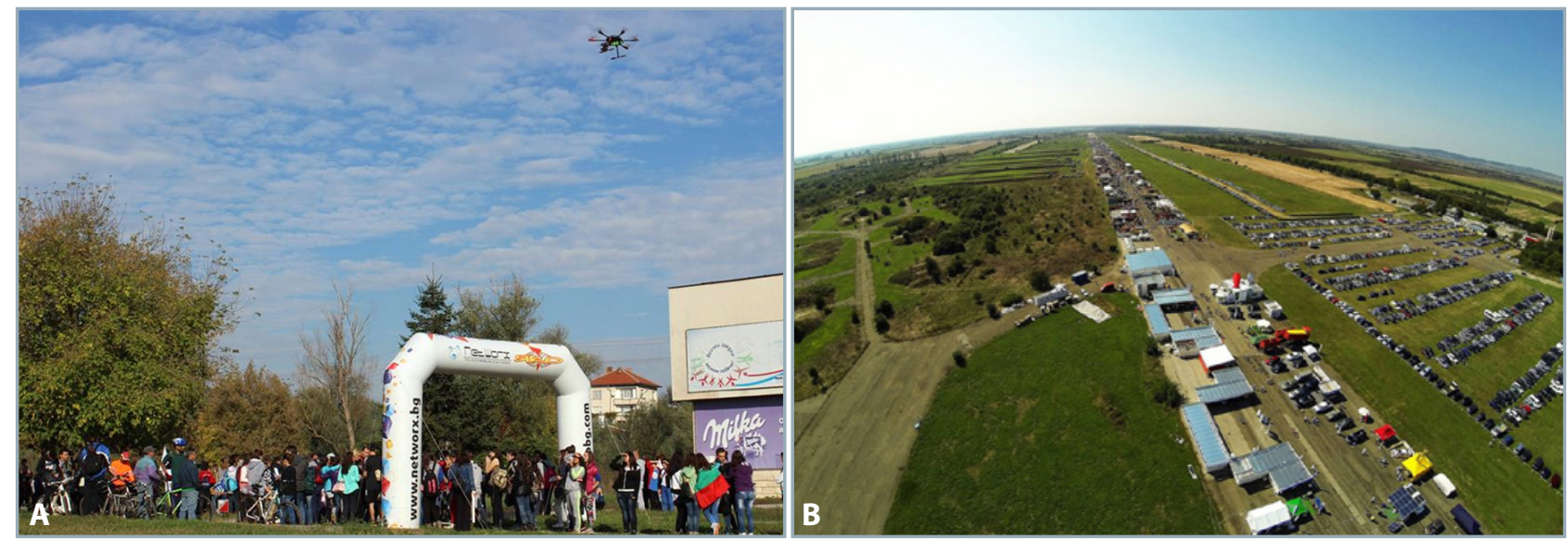

Figure 1A, B Examples of UAVs used for photography (A) or filming (B)

A - drone taking images during a sport competition; B - a scene from a commercial clip about an event (filmed by a fixed wing UAV)

Contact address: Ivan H. Beloev, University of Ruse "Angel Kanchev", Department of Transport, Ruse, Bulgaria e-mail: ibeloev@uni-ruse.bg 


\section{Aerial photography and filming using UAVS}

The majority of the modern UAVs are used by photographers for taking aerial photos during different events (including sport events, weddings, concerts, etc.). In some cases, the drones are also suitable for aerial filming of movie scenes, commercials, advertisement clips and so on. Some examples of the UAV usage are given above (Figure 1).

\section{UAVS for search and rescue operations, security, logistics and inspection}

Every second counts in search and rescue operations. In order to function as efficiently as possible, it is important to be able to obtain a rapid overview of the situation - the type of view that is often only possible from the air. While the planes and helicopters require some time to get ready for deployment, the UAVs can be put into action immediately and without any loss of time (DeBusk, 2009). The carbon fibre housing of the drones allows them to operate successfully under heavy conditions such as rain, snow or in extreme temperatures, including fire (Figure 2). With surveillance and video data being transferred in real time, it is no longer necessary for humans to enter the danger zone in order to analyse the situation - the UAVs can now can provide this instead (Doherty and Rudol, 2007; Barnard, 2010).

With large gatherings and protests or when monitoring single individuals, it is often difficult to maintain an overview. UAVs can be a useful instrument allowing the situation to be viewed from above. Considering surveillance, a high level of noise emitted by the helicopters and planes is perceived as disadvantage. The highly efficient electric motors developed especially for the UAVs allow the discrete and almost silent surveillance. In addition to this, with a dedicated link for high resolution video feed, the security operations can be coordinated in real time. Another great area for use of the surveillance UAVs is for border protection and homeland security (Haddal and Gertler, 2010). Equipped with infrared cameras, the drones have the potential to spot all trespassers or fugitives, making them a powerful weapon for customs and law enforcement officers.

The year 2013, during which a great number of new technologies was designed especially for the aerial vehicles, was ground-breaking for the UAVs. It also presented the next application level for unmanned aerial systems - the use of drones as Unmanned Cargo Systems. Dedicated

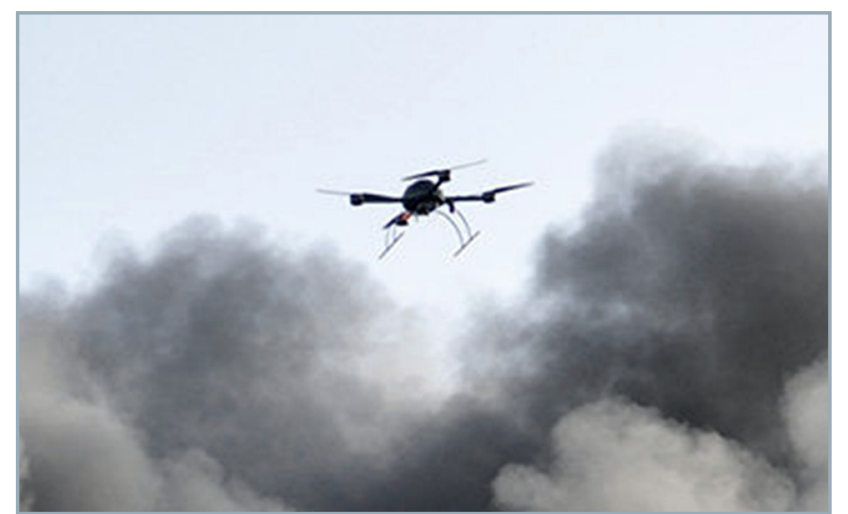

Figure 2 A multirotor UAV is being used for real-time aerial overview of fire

Source: www.microdrones.com teams of aircraft engineers and logistics experts focused on developing the industry standards for delivering parcels around the globe in a future of safe, fast and environmentally friendly transportation of goods involving a fraction of the cost compared to former standards. Based on GPS waypoint navigation, special medical drones were used to deliver urgent medicines (Figure $3 \mathrm{~A}$ ). Cargo drones were also used for delivering common goods (Figure $3 \mathrm{~B}$ ) to remote and hard-to-reach areas, such as in the mountains or over the sea, rivers and lakes.

To ensure the supply of electricity, gas and oil, the regular inspections of the installations are imperative. Because of their size, structure or location, they are often inspected from the air. These inspections can be carried out with the help of UAVs. Using the mission planning software, the UAVs can inspect the relevant installation autonomously, without the need for navigation instructions from an operator. The result is rapid, even though the inspection is performed in-depth. With an UAV, it is even possible to inspect high voltage power lines and wind turbines. Specialized UAVs are developed to withstand voltage up to $380 \mathrm{kV}$ and are unaffected by magnetic fields (Barnard, 2010).

The use of UAVs for inspection can involve oil and gas pipeline inspections, solar installation inspections, detection of the spread of algae, power line and cable inspections, cooling tower inspections, critical infrastructure inspections, wind turbine inspections, bridge inspections, counting of animal populations, train line inspections, monument inspections, radiation measurement and monitoring.
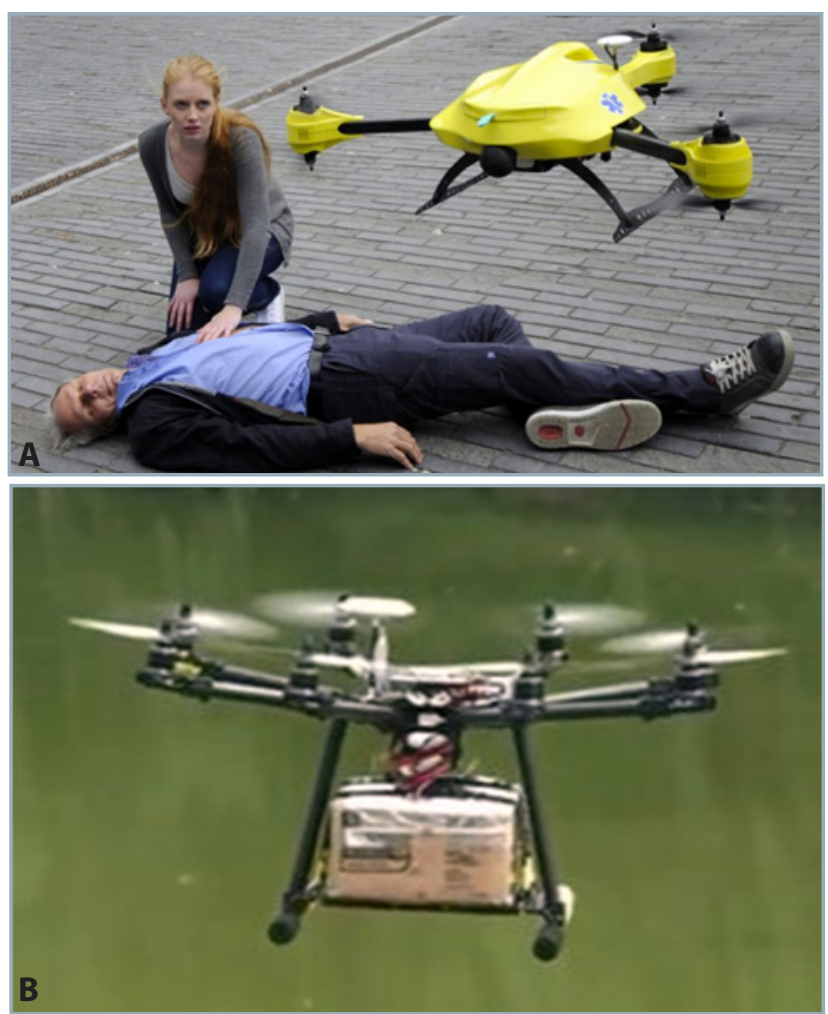

Figure 3A, B Examples of UAVs used for first-aid (A) and postal services (B)

A - medical multi-copter equipped with first-aid kit and defibrillator arriving at the scene of an incident; $B$ multirotor UAV for parcel deliveries and postal services Source: A - www.tudelft.nl; B - www.56.com 


\section{UAVS for GIS improvement and for land and water surveying}

The use of UAVs increases the efficiency of the GIS professionals by allowing them to capture accurate aerial images (Manyoky et al., 2011). These images can be later transformed into 2D orthomosaics/maps or into 3D models. This new method of raster data collection complements existing techniques, fitting between satellite or manned aircraft imagery and the time-consuming highly accurate terrestrial approaches. The UAVs allow the users to collect geo-referenced images without the need for booking and waiting for manned aircraft or satellite imagery. The planning of the UAV mission, the flight, the images acquisition and the processing of the data can all be accomplished in one day (Sarris, 2001). The Drones can achieve orthomosaic or 3D model accuracy of roughly 1 to 3 times the ground sample distance (or GSD - a term used in aerial photography, which presents the distance between pixel centres measured on the ground) or down to $4.5 \mathrm{~cm}$ per pixel, which is significantly higher than current satellite and manned aircraft imaging services. Additionally, the use of the unmanned aerial vehicles is much easier, since cloud cover is not an issue (Manyoky et al., 2011). Figure 4 represents an overview of an actual UAV flight pattern for a GIS mission using a specialized software and Figure 5 shows the post processing results of the acquired images (left figure is the generated orthomosaic and right figure is the digital elevation model (DEM)).

One of the major advantages of the drones for land and water surveying is the fact that they can vastly reduce the time for data collection. The acquiring of raster data from the air in the form of geo-referenced digital aerial images, which are of resolutions up to $1.5 \mathrm{~cm}$ per pixel, is a very simple and flexible process and can result in millions of data points in just one flight. Additional accuracy and time savings can be achieved by using a survey-grade UAV with RTK capabilities. The GNSS/RTK receiver systems allow the drone to receive real-time data corrections from a base station or via VRS and an absolute $X, Y, Z$ accuracy of down to $3 \mathrm{~cm}$ can be achieved with the help of this system, even without any Ground Control Points. This all makes the data collection very simple, but also very productive process. A final advantage point in

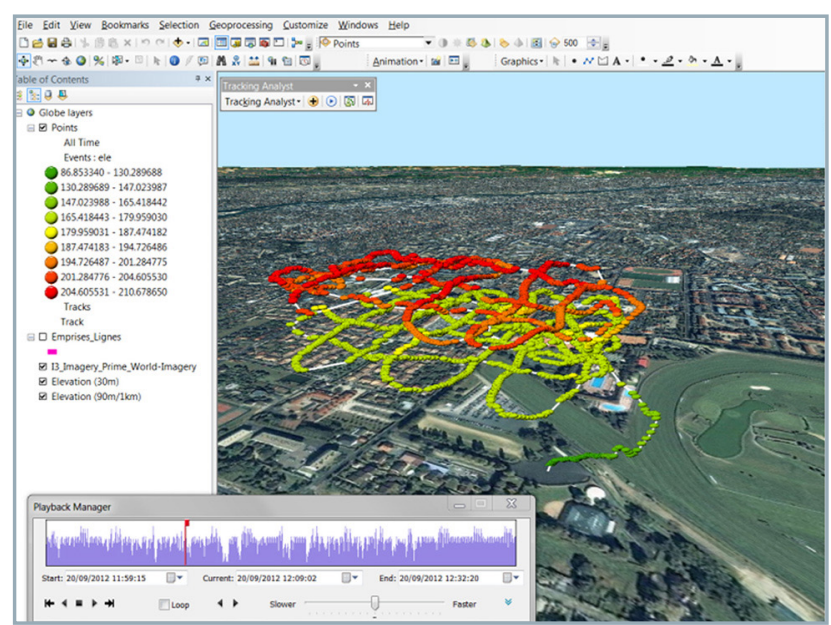

Figure 4 An overview of an actual UAV flight pattern for a GIS mission

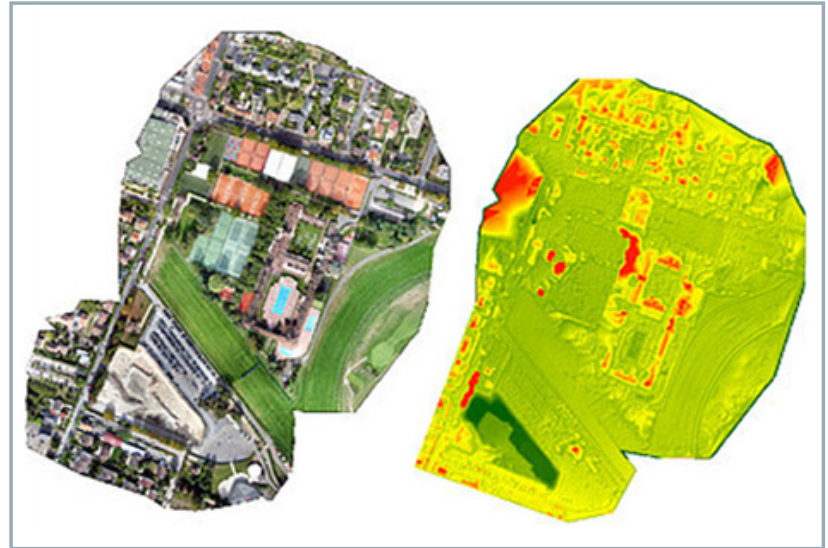

Figure 5 Orthomosaic and digital elevation model of the surveyed area

favour to the UAVs is that the support and operations crew have to spend the lesser amount of time on the ground, meaning additional minimizing of the risk to the surveying teams when measuring sites such as mines, unstable slopes and transport routes.

\section{UAVS for precision agriculture}

The precision technology has driven the farming revolution of the recent years, but the possibility to monitor the crops from the air has provided priceless information to the farmers and had increased their harvests significantly. With just a single flight of a specialized UAV, the farmer can receive dozens of highly accurate images from different fields with coverage of hundreds of hectares and all this without the cost and problems that usually comes with the use of manned services. In addition to this, the obtained images will be of far greater resolution than those provided by satellite services and they will not be influenced by the cloud cover (Sarris, 2001). By means of image processing software, the aerial photographs can be combined into one large orthomosaic image and thanks to specialized algorithms, like the Normalized Difference Vegetation Index (NDVI), the complete image and a reflectance map of the crops can be created. This map is the key to better yields and lower costs and will drive the agricultural business forwards. This technology highlights exactly which areas of crop need closer examination - meaning less time spent scouting and more time for treatment of the plants that need it. The images below (Figure $6 \mathrm{~A}$ and Figure $6 \mathrm{~B}$ ) are taken by UAV for precision agriculture equipped with a NIR camera. The image on the left is a colourful composite of all three bandsNIR, Green and Red. The image on the right is a processed Normalized Difference Vegetation Index (NDVI) image. These two images show how details and information, that is not visible to the naked eye and not visible in unprocessed multispectral imagery, can be extracted through processing and then used for taking the necessary actions in order to obtain better results and harvests.

The results, obtained after the processing of the images taken from the UAVs, like the ones in Figure 6, can be used to identify crop health and stress, and to identify problematic areas within the crops, which will allow an early targeted treatment. Plants need the appropriate levels of nutrients in order to thrive and produce a strong yield. The appropriate 

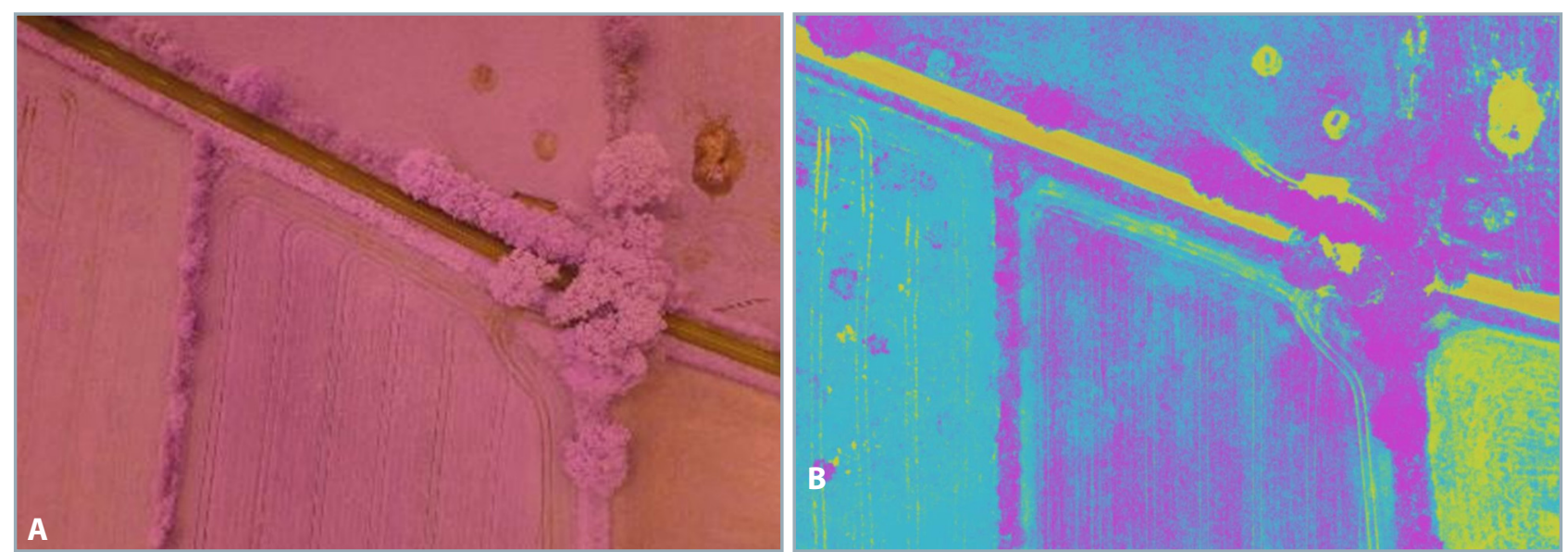

Figure 6A, B Different types of images, which are used for analysis of the current state of the yields for precision agriculture A - NIR image; B - NDVI image

levels of nitrogen will ensure strong growth of vegetation and foliage, appropriate levels of phosphorous are required for strong root and stem growth and appropriate levels of potassium are necessary for improving of the resistance to disease and also to ensure a better quality of crop. If soil lacks any any of these nutrients, the plant will become stressed and will struggle to thrive. NDVI Index mosaics offer the possibility to identify exactly which areas of the crops are stressed or struggling and to target directly these areas. The $\mathrm{NIR} /$ multispectral imagery provided by the UAVs can identify these management zones long before the problem become visible to the naked eye. This means that these management zones can be targeted before crop development and yield is affected.

\section{UAVS for monitoring of ecosystems and wildlife preservation}

Advance in aerial equipment and technology had made UAVs a key part of protection strategies of marine reserves, forests, deserts and many other landscapes. Drones are also widely used for remote observation of the habitats and the daily lifecycle of many endangered species. This all is possible with the help of many different sensor technologies in combination with a variety of cameras (Wezeman, 2011).
In forestry and nature protection, UAVs can be applied to many tasks, such as forest fire detection and damage assessment (Figure 7), illegal tree harvesting monitoring, trespassers monitoring, locating harvest sites and inspecting forestry operations, monitoring and change detection within hard-to-reach natural forests, determination of the biomass, vegetation growth and quality, control of invasive species, river erosion, deforestation, landslide risk assessment, etc.

Beside a thorough analysis of the images, many of the above mentioned applications require images to be processed and integrated as georeferenced and orthorectified products. There are several widely known methods for non-destructive detection of stress in trees - airborne chemical and bio-sampling, visual analysis, fluorescence measurements, optical reflectance measurements and thermal infrared evaluations and all of these methods can be performed using UAVs.

River obstructions, such as fallen trees, branches, dams or human garbage, can have significant impacts on water and sediment quality due to changes in flow patterns. These obstructions result in upstream flooding and in slower flows both upstream and downstream. These reduced flows and the dissipation of energy, as water seeps slowly through the obstruction, result in change of the environment
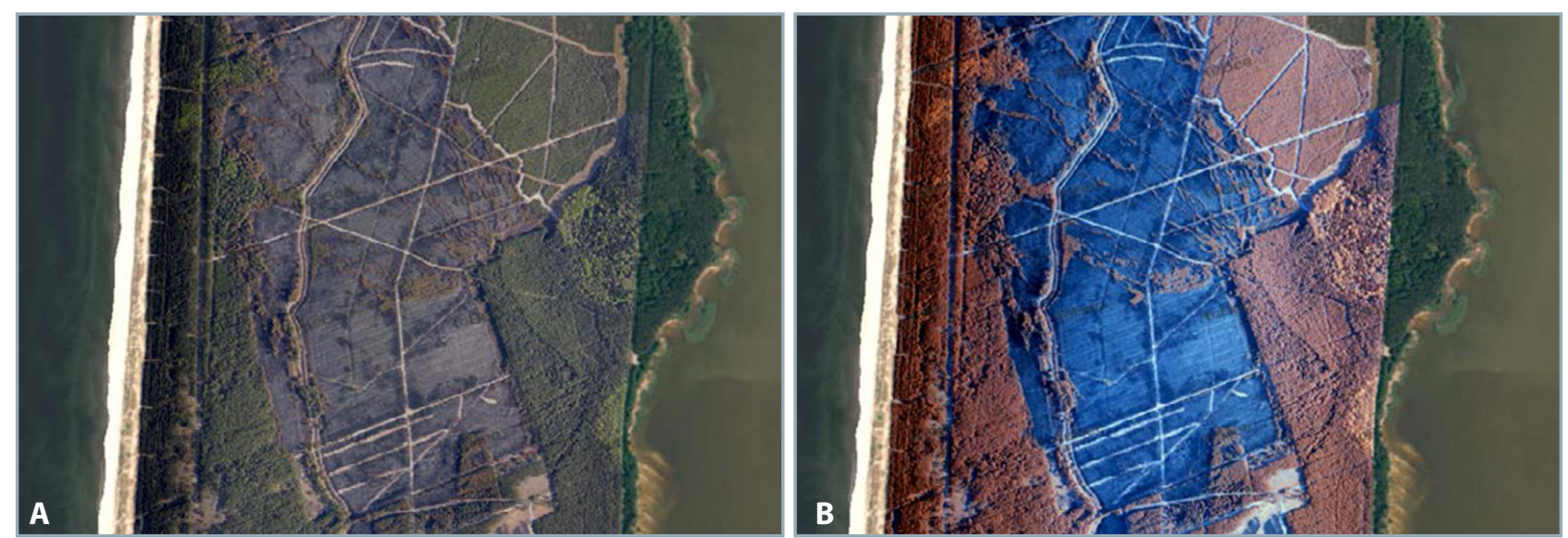

Figure 7A, B Examples of UAV images used for forest fire damage assessment

A - RGB orthomosaic generated from images acquired by UAV; B - NIR version of the same orthomosaic with red representing live vegetation and blue represents dead trees

Source: DeBusk, 2009 


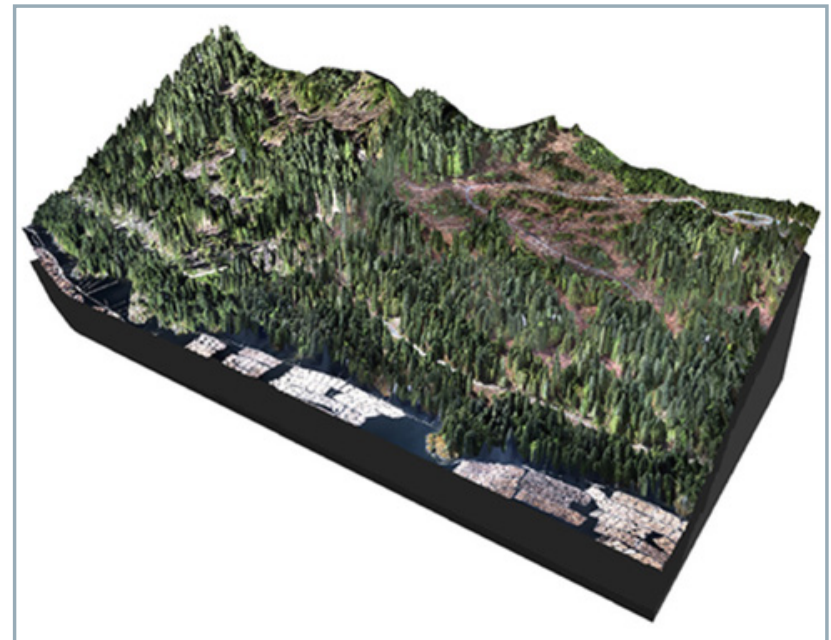

Figure 8 3D reconstruction of a creek and its surroundings Source: www.micromappers.wordpress.com

downstream and causes meandering and bank erosion upstream. River obstructions also tend to reduce or eliminate any pattern of regular inundation and deposition of nutrient-rich sediments on floodplains in favour of a more regular flow regime. Other potential impacts of river obstructions on water and sediment quality might include changes in the sediment load, in the water temperature and in the chemical characteristics.

As obstruction flows slowly down the upstream of a river, the bedload (sediment carried along the river bed), suspended sediment, and organic material settle to the bottom, changing the character of the sediments. The settling of sediments can result in lower concentrations of suspended sediment downstream of the obstruction and the loss of gravel and sand bars.

Slower-moving water upstream and downstream of the obstructions can warm more than natural river flow due to longer sun exposure. Deep reservoirs or ponds upstream of the obstruction can become stratified.

The upstream flooding that results from river obstructions can lead to a surge of nutrients and other chemical compounds, released from soils and the decomposition of drowned vegetation in flooded areas. This can potentially lead to high biological productivity, which can persist for varying lengths of time, depending on the amount of flooded vegetation, soil composition, and basin characteristics. However, high biological productivity can consume oxygen, potentially leading to low oxygen levels in bottom waters and the conversion of chemicals

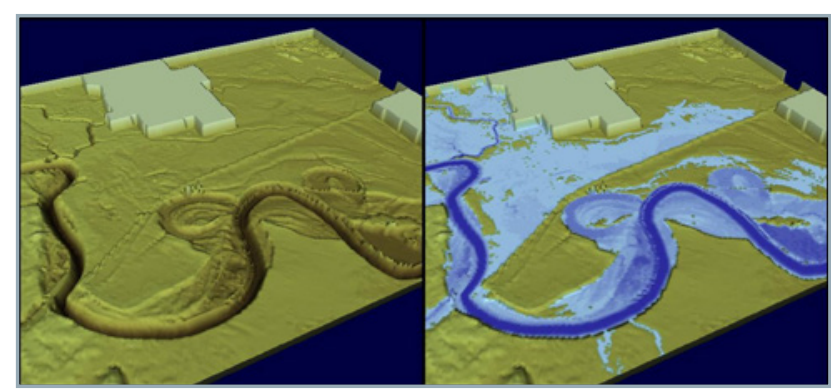

Figure 9 Simulation of river flooding using 3D terrain model obtained from UAV images Source: Lei Ma et al., 2013 between different forms (e.g., the reduction of nitrate, $\mathrm{NO}_{3}^{-}$, to ammonium ion, $\mathrm{NH}_{4}^{+}$, under conditions of low oxygen). In some areas, mobilization of mercury in reservoirs and flooded areas can lead to increased mercury contamination of fish.

All of this defines the inspection of the rivers and the maintenance of the riverbeds as very important tasks, which should be performed on a regular basis. Regardless of their importance, these processes are very time consuming and often require well trained and properly equipped ground teams. In order to reduce costs, to increase productiveness and to save time, an UAV can be used for inspection of the rivers and the riverbanks. Using modern mission planning software, the drone can be set to follow waypoints along the river while at the same time it can film surroundings or take pictures of it. Additionally, an overall high altitude overview of the entire area can also be obtained by slowly moving or gliding fixed-wing UAV. Then, the overview images can be processed and presented in the form of an aerial orthomosaics. By having the orthomosaics geotagged, they can easily be used for a 3D reconstruction of the area creation (Figure 8) or a digital terrain model, which could help pinpoint the places with obstructions and could provide valuable information to the river maintenance personal.

River obstructions are not the only causes of flooding. Excessive amounts of rain, overflowing dams or natural disasters can also lead to such events. By means of specialized software and usage of the 3D terrain models of the river area, a simulation of the potential impact of the flooding can be performed, which can be used to prevent damage or even save lives (Figure 9).

Preservation of the flora and the fauna has always been an important process, especially if it involves endangered species. Similar to the precision agriculture, the NIR and the NDVI images from the UAVs can be used for monitoring of the vegetation state in a given region (Figure 10). Based on these images, and without any need for additional ground inspection of the region, the necessary measures for preserving specific sorts of plants, bushes or trees could be applied in a much easier and much faster manner. The NDVI images can also be used for localization of invasive species, which would allow to make a much earlier response to their spreading and would allow to battle against them far more efficiently.

UAVs can also play important role in observation or monitoring of wildlife (Figure 11). Thanks to the modern technologies, the UAVs are producing less noise, compared to aeroplanes or helicopters, and will cause less disturbance or stress to the animals.

\section{Archaelogy and preservation of the national heritage using UAVS}

The idea of using UAVs equipped with video cameras and artificial intelligence in archaeology is a relatively new trend (Project Archaeocopter, http://www.archaeocopter. de/). Cultural resources such as archaeological sites and old buildings are under incessant pressure from property development and business organizations. While resources for heritage management and protection have been stagnant at best for a long time, technological development 

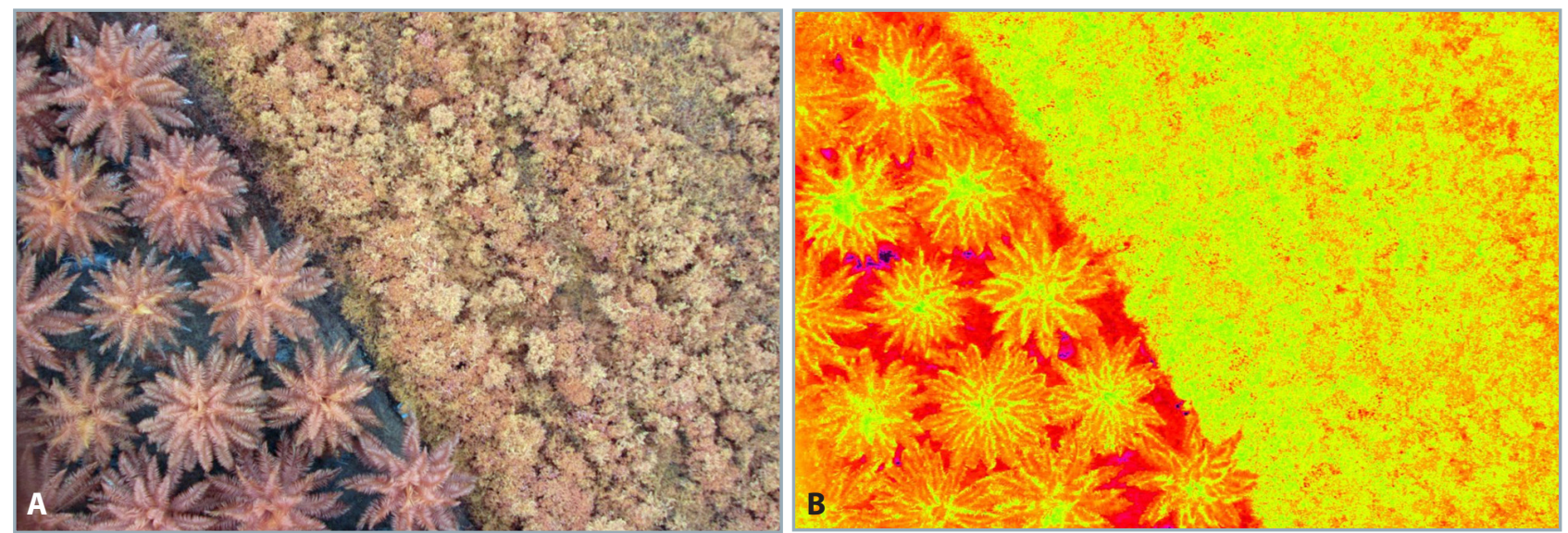

Figure 10A, B Raw (A) and NDVI (B) images for monitoring of invasive vegetation species $A$ - raw image; $B$ - NDVI image Source: Lei Ma et al., 2013
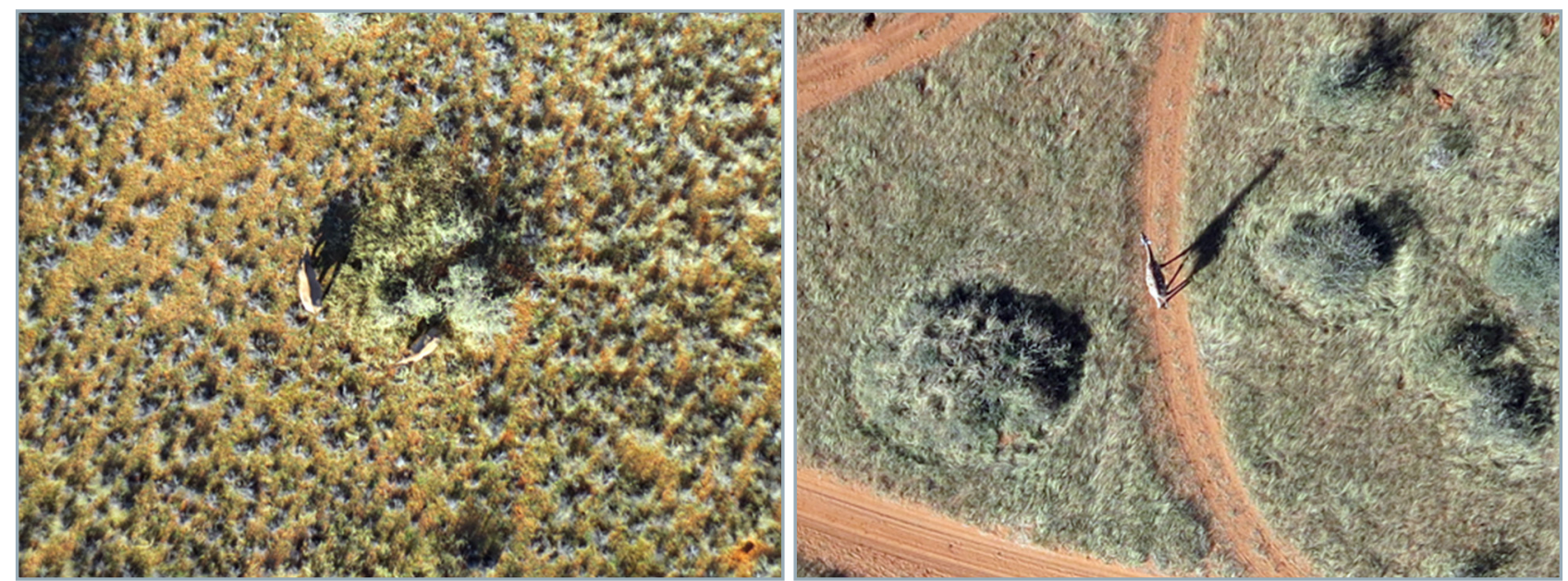

Figure 11 High altitude UAV images of free roaming wildlife Source: Blyenburgh et al., 2011
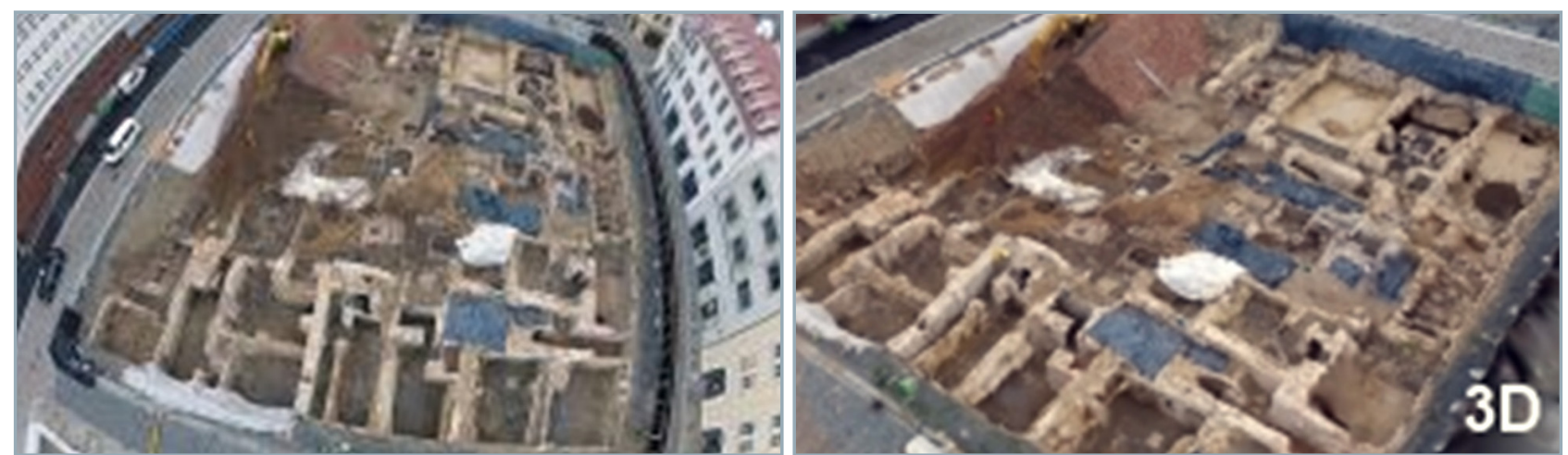

Figure 12 UAV photographs can be used for 3D reconstruction of archaeological sites Source: http://www.archaeocopter.de

in image-based 3D reconstruction have been fast-paced, especially in the field of computer vision. At the same time, robustness and affordability of ultralight UAVs have reached a point where this type of hardware can become an integrated part of the standard toolset for archaeological site recording.

The acquisition of high-resolution, georeferenced imagery is a fundamental prerequisite for the detection and documentation of archaeological heritage. Structure from
Motion (SFM) is a popular and robust method for producing 3D reconstructions from series of overlapping images (multi-view reconstruction) (Project Archaeocopter, http:// www.archaeocopter.de/). It provides heritage professionals with a flexible and low-cost method for the documentation and digital preservation of objects of interest, especially complex architectural remains (Figure 12). With the help of near real-time sparse 3D reconstruction, the operator of the UAV can immediately validate the data quality and the 


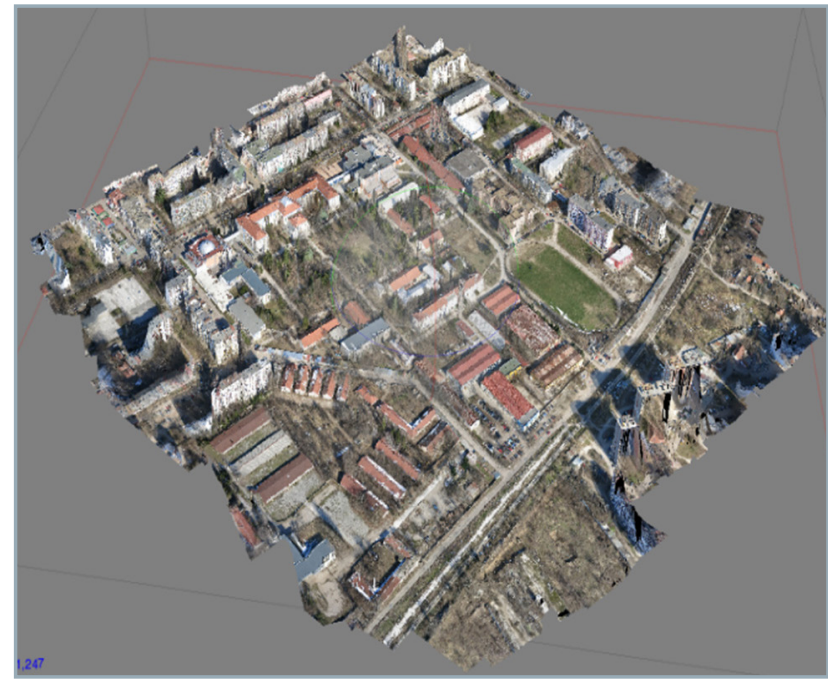

Figure $13 \quad 3 \mathrm{D}$ reconstruction model of the University of Ruse campus

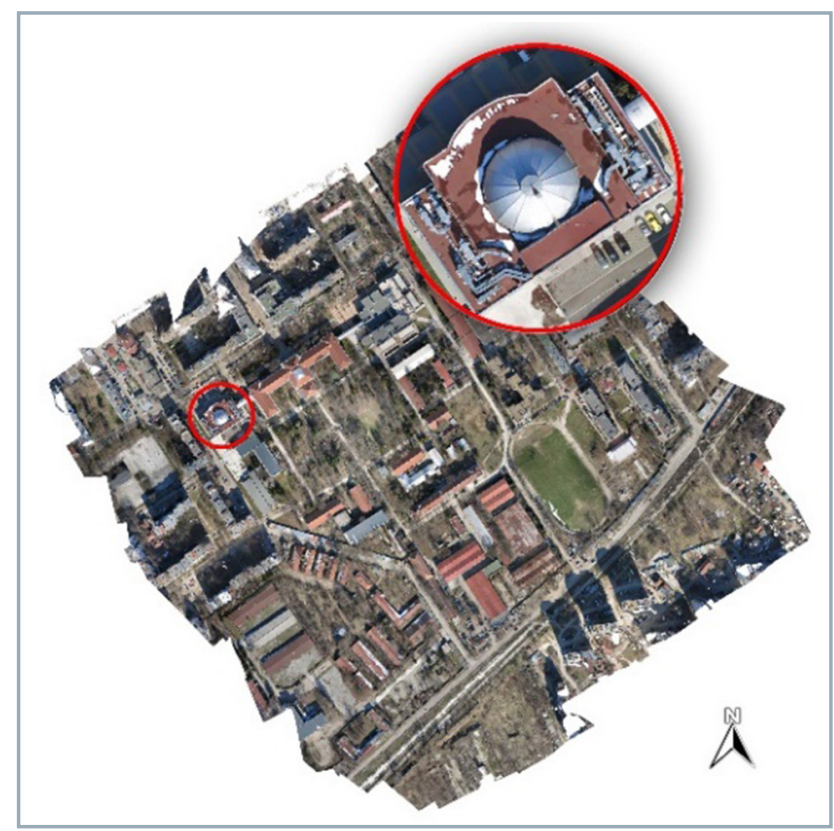

Figure 14 Orthophoto image with very high resolution of the University of Ruse campus

density of the input. Post processing steps include detailed surface reconstruction and texturisation of the triangulated point cloud, as well as cleaning and simplification of the final triangulated models. Examples of ground and aerial photographs, which are used for the 3D reconstructions of objects and archaeological sites are given above.

Preservation of the national heritage is a very important mission for every nation. UAVs can play an important role in this process by providing means for the creation of aerial photographs and films of the objects with great significance. Additionally the aerial photographs could be used for the creation of high quality 3D models of the objects (Figure 13 and Figure 14).

\section{Conclusion}

The technological advance had made the unmanned aerial vehicles much more affordable and a way easier to operate.
This has offered new opportunities for the drone applications and they are currently involved in many activities - from taking photographies and video recordings of events to inspections of dangerous installations and buildings, tracking of trespassers or delivering of parcels and mails. Lately, the UAVs are being also actively used for tasks, which were considered unthinkable just few years ago. Some of these activities involve 3D reconstruction of objects, precision agriculture, animal tracking and archaeology.

The application possibilities of the drones are unlimited, but nevertheless, there are still many challenges to be solved. The average flight time of the UAVs is still under one hour and the payload of the majority of the drones is still measured in grams. These challenges, as well as other, still represent major issues for the wider use of the UAVs, but with their potential now being fully understood, it is only a matter of time before solutions to the challenges will be provided and the drones will be used in all possibly imaginable fields and applications.

\section{References}

BARNARD, J. 2010. Remotely Piloted Aircraft in oil, gas and mineral exploration and production activities. In Presentation at the Royal Aeronautical Society Conference'Towards commercial exploitation of Unmanned Aircraft', London, November 11. 2010.

BLYENBURGH, P. et al. 2011. 2011/2012 UAS Yearbook - UAS: The Global Perspective. 9th Edition. Grand Forks, ND: UND.

DEBUSK, W. M. 2009. Unmanned Aerial Vehicle Systems for Disaster Relief: Tornado Alley. Atlanta : Georgia Institute of Technology.

DOHERTY, P. - RUDOL, P. 2007. A UAV Search and Rescue Scenario with Human Body Detection and Geolocalization. In Lecture Notes in Computer Science, vol. 4830, ed. M.A. Orgun and J. Thornton, pp. 1-13. New York : Springer.

HADDAL, C. C. - GERTLER, J. 2010. Homeland Security: Unmanned Aerial Vehicles and Border Surveillance. In Congressional Research Service Report for Congress. Washington. July 8, 2010.

Images courtesy of MICRODRONES GMBH and acquired from www. microdrones.com

Images courtesy of Alec Momont and TU Delft - acquired from www.tudelft.nl

Images courtesy by and acquired from www.56.com

Images courtesy by Micromappers - micromappers.wordpress.com LEI MA et al. 2013. Using unmanned aerial vehicle for remote sensing application. In Proceedings of the 21st International Conference on Geoinformatics (GEOINFORMATICS), Kaifeng, China, 2013.

MANYOKY, M. - THEILER, P. - STEUDLER, D. - EISENBEISS, H., 2011. Anwendung von UAV's in der Katastervermessung, cadastre, 5 (April 2011), pp. 16-17.

Project Archaeocopter - the Flying archaeologist at http://www. archaeocopter.de/

SARRIS, Z. 2001. Survey of UAV applications in civil markets. Chania, Greece: Technical University of Crete, Department of Production Engineering and Management. Retrieved from: http://med.ee.nd. edu/MED9/Papers/Aerial_vehicles/med01-164.pdf. Accessed January 4, 2011.

WEZEMAN, S. UAVs and UCAVs: Developments in the European Union. Briefing Paper for the Eurepean Parliament. Retrieved from: http://www.sipri.org/research/armaments/transfers/publications/ other_sipri_publ/20071000. Accessed February 4, 2011. 